\title{
Virtual Screening of Penicillin-derived Inhibitors for the Metallo- $\beta$-lactamase from Bacillus cereus
}

\author{
Jong-Sun Lee, ${ }^{\dagger}$ Ethan White, ${ }^{\dagger}$ Sang Gon Kim, ${ }^{\dagger}$ and Sung-Kun Kim ${ }^{\dagger, *, *}$ \\ ${ }^{\dagger}$ Department of Chemistry and Biochemistry, ${ }^{\dagger}$ the Institute of Biomedical Studies, Baylor University, Waco, \\ Texas 76798-7348,USA. *E-mail: sung-kun_kim@baylor.edu \\ Received August 30, 2010, Accepted October 6, 2010
}

\begin{abstract}
The metallo- $\beta$-lactamases (M $\beta$ Ls) are clinically significant enzymes which readily hydrolyze most $\beta$-lactam antibiotics. Discovering potential inhibitors for the $\mathrm{M} \beta \mathrm{Ls}$ is an expensive, time consuming endeavor. Virtual screening can sieve out inhibitor candidates with incompatible features prior to synthesis, decreasing these costs. Using Autodock 4.0, the binding locations and energies of four previously-studied potential inhibitors and four additional compounds obtained from the National Cancer Institute (NCI) database were computationally calculated. Based on the docking models of these eight compounds, we then designed several hypothetical inhibitor structures, compounds A through F, and performed their respective docking experiments. The docking results for compound $\mathrm{F}$ showed that it binds to the zinc containing active sites with a lowest predicted binding energy of $-6.70 \mathrm{kcal} / \mathrm{mol}$, suggesting $\mathrm{F}$ is the most likely potential $\mathrm{M} \beta \mathrm{L}$ inhibitor.
\end{abstract}

Key Words: Metallo- $\beta$-lactamase, Bacillus cereus, Inhibitor, Virtual screening, AutoDock 4.0

\section{Introduction}

$\beta$-Lactam compounds have successfully carried out their role as antibiotics by inducing bacteriolysis. ${ }^{1,2}$ However in recent decades, bacteria have evolutionarily developed abilities to counteract $\beta$-lactam containing antibiotics by producing $\beta$ lactamases, ${ }^{3}$ which catalyze the cleavage of the $\mathrm{C}-\mathrm{N}$ bond in the $\beta$-lactam ring, thereby allowing bacteria to resist the remedial effects of this family of antibiotics. ${ }^{4,5}$ The $\beta$-lactamases have been classified into four different groups, A through $\mathrm{D}$, based on their structures and catalytic mechanisms. ${ }^{6}$ In group A, C, and D $\beta$-lactamases, an active site serine nucleophilicily attacks the carbonyl group of the $\beta$-lactam ring, while group B enzymes, commonly referred to as metallo- $\beta$-lactamases ( $\mathrm{M} \beta \mathrm{Ls})$, utilize zinc ions for catalytic activity. ${ }^{7-9}$

The $\mathrm{M} \beta$ Ls have been noted as clinically significant zinc hydrolases because they readily hydrolyze the majority of $\beta$ lactam antibiotics. ${ }^{10} \mathrm{~A}$ zinc ion in the active site acts as an electron pair acceptor, catalyzing the hydrolysis of the $\beta$-lactam ring in various classes of antibiotics, such as penicillins and cephalosporins, and even in a group of serine-based $\beta$-lactamaseresistant antibiotics, carbapenems. ${ }^{11,12}$ Several studies have suggested potential inhibitors that either emulate the structure of the $\beta$-lactam antibiotics or interact with the zinc binding site. ${ }^{13}$ Inhibitors containing penicillin scaffolds are particularly attractive because they can compete with $\beta$-lactam antibiotics for the $\mathrm{M} \beta$ Ls active site. However, though many potential M $\beta$ Ls inhibitors have been proposed, none have yet shown clinical usefulness. Therefore, research efforts should still be focused on discovering more effective inhibitors.

Designing enzyme inhibitors requires significant amounts of time and laboratory resources. Because only five out of every 40,000 synthesized inhibitors that undergo animal testing actually make it to clinical trials and typically only one of those five are approved, ${ }^{14}$ applications of computational techniques are becoming indispensible in the process of drug discovery and development. Virtual screening, or in silico modeling, automatically sieves out compounds with incompatible features. Consequently, the portion of time and financial resources spent on fruitless experimental synthesis can be significantly decreased. ${ }^{14-16}$

AutoDock is a molecular modeling program which has been previously used for designing inhibitors. ${ }^{17-20}$ It predicts the docking of flexible small molecules, or drug candidates, to receptors of enzymes and macromolecules. This program also provides a free-energy force field to evaluate docking conformations and binding energies during the docking simulations. Free-energy scoring is based on a linear regression analysis which calculates electrostatic energy, desolvation energy, the energy in hydrogen bonding interactions, and entropy.

In this report, we describe the application of structure-based virtual screening for the evaluation and optimization of several penicillin-derived inhibitor candidates of Bacillus cereus $\mathrm{M} \beta \mathrm{L}$ using AutoDock 4.0. Based upon the docking data of the optimized compounds, we then suggest a novel potential M $\beta \mathrm{L}$ inhibitor for future investigation. Since these computational experiments are conducted using freely available software, the in silico research strategy demonstrated here can be utilized for inhibitor discovery.

\section{Methods}

Preparation of target protein structure. The 3D structure of $\mathrm{M} \beta \mathrm{L}$ from Bacillus cereus was obtained via the protein data bank (PDB entry 1BC2) (http://www.pdb.org/pdb/explore/explore.do?structureId=1BC2). The enzyme has been determined at $1.9 \AA$ resolution in crystal form to have two identical chains in the asymmetric unit. Each chain contains two zinc ions in the active sites. $\mathrm{Zn} 1$ is tightly coordinated by His86, His88, and His149, while Zn2 is loosely coordinated by Asp90, Cys- 
(a)

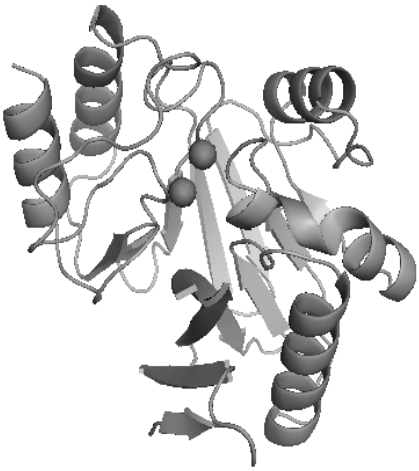

(b)

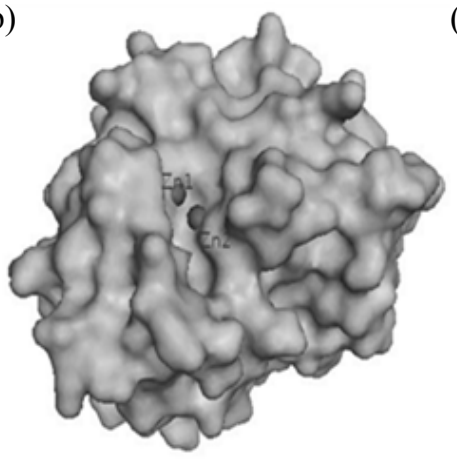

(c)

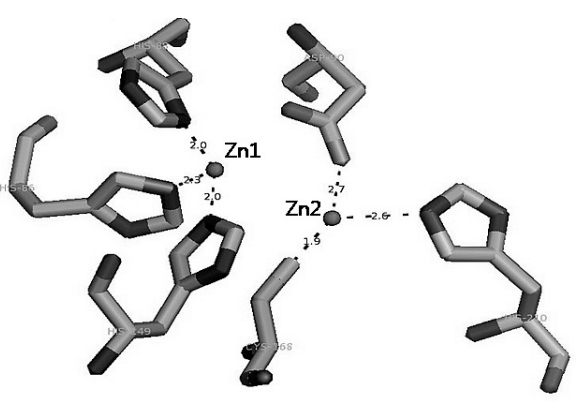

Figure 1. The structure of the zinc-dependent $\beta$-lactamase II from Bacillus cereus. (a) A ribbon structure of the Bacillus cereus, with the two zinc ions. (b) A molecular surface map of the Bacillus cereus, with the $\mathrm{Zn} 1$ and $\mathrm{Zn} 2$ active site binding pocket. (c) Zincs active site geometry with distance between zinc ion and coordinated residues.

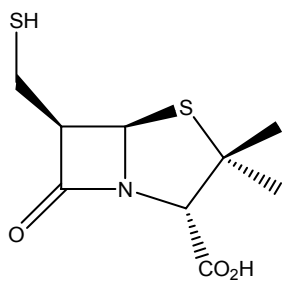

$5 \mathbf{a}$

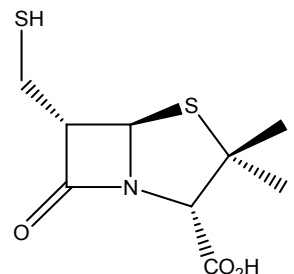

$5 \mathbf{b}$

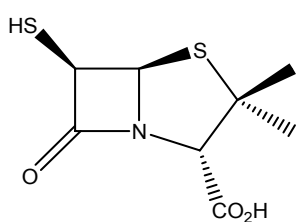

$6 a$

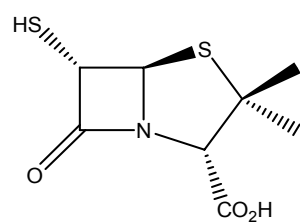

6b

Figure 2. Previously suggested $\beta$-lactamases inhibitors.

168, and His210.22 All atom parameters were automatically created by AutoDockTools (ADT). Coordinates for the 1BC2 were processed in ADT by adding hydrogen atoms, assigning charges with the Gasteiger method, and merging the non-polar hydrogens. We modified $1 \mathrm{BC} 2$ by removing one of the chains using ADT because AutoDock 4.0 only allows one target molecule. The 3D structure, the topology of the zinc active sites, and the bonding distances between the zinc ions and active site residues were confirmed by PyMol (Figure 1).

Docking with predefined compounds. To increase the validity of potential inhibitor and docking studies, previously-studied (predefined) inhibitor candidates taken from literature were chosen as control dockings. ${ }^{23}$ All the structures of the compounds and their inhibitory activities are shown in Figure 2 and Table 1. Next, we utilized the Dundee PRODRG[2] server to generate a topological description of the inhibitor candidates (http://davapc1.bioch.dundee.ac.uk/programs/prodrg/prodrg. $\mathrm{html}){ }^{24}$ This server converted 2D compounds drawn by the JME editor to 3D coordinates in PDB format, adding hydrogen atoms. The 3D structures of the compounds, in PDB format, were then docked with the $1 \mathrm{BC} 2$ monomer using AutoDock4.0 (http:// autodock.scripps.edu). In order to allow for the flexibility of the active site, His 86 of the protein was selected and given one active torsion. A grid map of $62 \AA \times 62 \AA \times 62 \AA$ points with $0.375 \AA$ grid spacing was centered on the zinc active sites and was calculated around the docking area by running AutoGrid. The Lamarckian genetic algorithm was used as the search method, and the docking parameters were set to 100 automated docking runs for a 150 population size with a 2,500,000 maximum number of energy evaluations for each docking experi-
Table 1. $\beta$-Lactamase inhibitory activity $\left(\mathrm{IC}_{50}, \mu \mathrm{M}\right)$ against representative class A (TEM-1), class C (P99), and class B (metallo) enzymes

\begin{tabular}{cccccc}
\hline Compound & $\begin{array}{c}\text { TEM-1 } \\
\text { (Class A) } \\
\text { Serine }\end{array}$ & $\begin{array}{c}\text { P99 } \\
\text { (Class C) } \\
\text { Serine }\end{array}$ & $\begin{array}{c}\text { L1 } \\
\text { (Class B) } \\
\text { Metallo }\end{array}$ & $\begin{array}{c}\text { BCII } \\
\text { (Class B) } \\
\text { Metallo }\end{array}$ & $\begin{array}{c}\text { Ref\# } \\
\text { 5a }\end{array}$ \\
$\mathbf{5 b}$ & 601 & 0.10 & 32.1 & 2.9 & 23 \\
$\mathbf{6 a}$ & $>1000$ & 3.75 & 10.9 & 1.7 & 23 \\
$\mathbf{6 b}$ & 988 & 72.3 & 41.4 & 17.8 & 23 \\
& & & 36.9 & 17.2 & 23 \\
\hline
\end{tabular}

ment. The results showed the binding energies and hydrogen bond interactions between the ligands and the $1 \mathrm{BC} 2$ monomer, and the cluster analysis was performed based on root mean square deviation (RMSD).

NCI compounds screening. The Enhanced NCI (National Cancer Institute) Database Browser (http://129.43.27.140/ ncidb2/) screened for potential M $\beta$ L inhibitor structural motifs via PASS (Prediction of Activity Spectra for Substance) predicting activities, applying the queries "antibiotics penicillinlike" and " $\beta$-lactamases inhibitor." The browser carried out a similarity search of at least $95 \%$ Tanimoto coefficient similarity based on the penicillin scaffold. The Dundee PRODRG[2] server generated the selected compounds' 3D structures in PDB format and Autodock 4.0 docked the compounds to modified $1 \mathrm{BC} 2$. The Lamarckian genetic algorithm search methods and docking parameters from the predefined compounds docking experiments were used for docking screened compounds into the protein.

Potential new compound design and its docking. Maintaining 
a penicillin scaffold, we hypothetically designed new compounds based on the best binding results from the four predefined compounds and the compounds obtained from the NCI database. The hypothetical compounds were sketched with the JME editor and their coordinates were generated by Dundee PRODRG[2] server in PDB format. A docking experiment was conducted using the same search methods, the same docking parameters, and the same $1 \mathrm{BC} 2$ monomer as the target enzyme.

\section{Results and Discussion}

Overall procedure. To further a previous in vitro M $\beta \mathrm{Ls}$ inhibitor study via in silico methods, we generated 3D structures of the predefined M $\beta$ Ls inhibitors and obtained a 3D structure of the target protein $\mathrm{M} \beta \mathrm{L}$ from Bacillus cereus through the protein data bank (PDB). Four compounds were added to our study through screening of penicillin-like antibiotics (Figure 2) and $\beta$-lactamases inhibitors by PASS prediction with a similarity search performed in the Open NCI database (Table 3a and 3b). Computational experiments were performed to predict the docking conformations of the four predefined compounds and the four NCI compounds with the M $\beta \mathrm{L}$. Their predicted binding energies, which permit us to understand the overall binding energy between a ligand and the target protein, and the enzymeligand hydrogen bonding interactions were analyzed by AutoDock 4.0. Based on the results from our docking experiments with the eight compounds, we then designed several hypothetical M $\beta$ Ls inhibitor compounds. Lastly, we conducted docking experiments on these novel structures with the target protein and identified the most promising potential inhibitor.

Docking results with predefined compounds. A previous in vitro study suggested four penicillin-derived inhibitor compounds on which the hydroxyl groups were substituted for thiol groups. These compounds inhibit both representative metalloand serine- $\beta$-lactamases ${ }^{23}$ (Figure 2). Particularly, compounds $\mathbf{5 a}$ and $\mathbf{5 b}$ had the ability to selectively and actively inhibit the $\mathrm{M} \beta \mathrm{L}$ from Bacillus cereus (BcII), whose mechanistic and structural information has been elucidated in previous M $\beta \mathrm{L}$ studies. ${ }^{25}$ Compound $\mathbf{5 b}$ had the best BcII inhibitory activity among the four suggested compounds, with an $\mathrm{IC}_{50}$ value of $1.7 \mu \mathrm{M}^{23}$ (Table 1).

To confirm the results of the in vitro study via in silico modeling, the first docking experiments were carried out using the predefined compounds as ligands and the PDB entry 1BC2 as the target protein. The results of the AutoDock docking experiments are shown in Table 2. AutoDock4.0 designated similar binding positions for all four compounds. Their docking positions were located in the vicinity of the $\mathrm{Zn} 1$ active site (His86, His88, and His 149 residues). For compound 5a, the lowest predicted binding energy was $-6.37 \mathrm{kcal} / \mathrm{mol}$. Three hydrogen bond interactions occurred between the carbonyl oxygen of 5a's thiazolidine ring and the amine hydrogen of Lys 117 (1.885 $\AA$ ), the carbonyl oxygen of 5a's $\beta$-lactam ring and the amine hydrogen of Asn118 (1.808 $\AA$ ), and 5a's thiol group and the side chain of Asp152 (2.213 $\AA$ ). For compound $\mathbf{5 b}$, the lowest predicted binding energy was $-6.56 \mathrm{kcal} / \mathrm{mol}$. Three hydrogen bond interactions occurred between the carbonyl oxygen of $\mathbf{5 b}$ 's $\beta$-lactam ring and the imidazole side chain of His88 (2.015 $\AA$ ), the car- bonyl oxygen of 5b's thiazolidine ring and the amine hydrogen of Lys 117 (1.762 $\AA$ ), and 5b's thiol group and the side chain of Asp152 (1.778 $\AA$ ). For compound 6a, the lowest predicted binding energy was $-5.34 \mathrm{kcal} / \mathrm{mol}$. Two hydrogen bond interactions occurred between the carbonyl oxygen of 6a's $\beta$-lactam ring and the hydroxyl of Thr108 (1.799 $\AA$ ) and the carbonyl oxygen of 6a's thiazolidine ring with the amine hydrogen of Ala $109(1.840$ $\AA$ ). For compound $\mathbf{6 b}$, the lowest predicted binding energy was $-6.35 \mathrm{kcal} / \mathrm{mol}$. Two hydrogen bonds occurred between the carbonyl oxygen of 6b's thiazolidine ring and the $\varepsilon$ amino group hydrogen of Lys117 (2.019 ̊), and the carbonyl oxygen of $\mathbf{6 b}$ 's $\beta$-lactam ring and the amide hydrogen in the carboxamide side chain of Asn 118 (1.866 $)$ ),

The docking experiments show similar binding positions for all four compounds near the $\mathrm{Zn} 1$ active site. Since there is hydrogen bonding with His 88 , an active site residue coordinated to $\mathrm{Zn1}$, we expect $\mathbf{5 b}$ is more likely to obstruct substrate binding, either sterically or electronically, than the other three compounds. Figure 3 shows the best mode of 5b's docking interaction with the $\mathrm{M} \beta \mathrm{L}$ from Bacillus cereus. In addition, $\mathbf{5 b}$ has the lowest binding energy of the four compounds. Thus, our computational data is in accordance with the findings of a previous study where the $\mathrm{IC}_{50}$ value obtained with $\mathbf{5} \mathbf{b}$ was the lowest of the four tested compounds.

NCI database screening and docking results. As mentioned above, a previous in vitro study synthesized four penicillinderived compounds which were found to inhibit a representative $\mathrm{M} \beta \mathrm{L}$. However, based upon an earlier study, ${ }^{26}$ the inhibition pattern assay of the compound $\mathbf{5 b}$ revealed that the inhibition is reversible, noncompetitive. This observation is in good agreement with our computation analysis, where the binding site for compound $\mathbf{5 b}$ is not in the active site (Fig. 4). This prompted us to modify the design of compound $\mathbf{5 b}$ in an effort to obtain a robust competitive $\mathrm{M} \beta \mathrm{L}$ inhibitor.

The purpose of screening NCI compounds is to identify chemical moieties which may improve the design of compound $\mathbf{5 b}$. Given that compound $\mathbf{5 b}$ is primarily composed of a penicillin-like scaffold, we conducted a PASS search in the Enhanc-

Table 2. Docking results with predefined compound

\begin{tabular}{|c|c|c|c|c|c|}
\hline Compound & Ntor & $\begin{array}{c}\Delta \mathrm{G}_{\mathrm{AD} 4} \\
(\mathrm{kcal} / \mathrm{mol})\end{array}$ & $\begin{array}{c}\mathrm{RMSD} \\
(\AA)\end{array}$ & $\begin{array}{l}\text { H-bonding } \\
\text { interaction }\end{array}$ & $\begin{array}{c}\text { H-bond } \\
\text { distance } \\
(\AA)\end{array}$ \\
\hline \multirow{3}{*}{$5 a$} & \multirow{3}{*}{3} & \multirow{3}{*}{-6.37} & \multirow{3}{*}{10.58} & $\mathrm{CO} \cdots \mathrm{HN}($ Lys 117$)$ & 1.885 \\
\hline & & & & $\mathrm{CO} \cdots \mathrm{HN}(\mathrm{Asn} 118)$ & 1.808 \\
\hline & & & & SH...OC(Asp152) & 2.213 \\
\hline \multirow{3}{*}{$5 \mathbf{b}$} & \multirow{3}{*}{3} & \multirow{3}{*}{-6.56} & \multirow{3}{*}{0.688} & $\mathrm{CO} \cdots \mathrm{HN}(\mathrm{His} 88)$ & 2.015 \\
\hline & & & & $\mathrm{CO} \cdots \mathrm{HN}(\operatorname{Lys} 117)$ & 1.762 \\
\hline & & & & SH‥OC(Asp152) & 1.778 \\
\hline \multirow{2}{*}{$6 a$} & \multirow{2}{*}{2} & \multirow{2}{*}{-5.34} & \multirow{2}{*}{2.416} & $\mathrm{CO} \cdots \mathrm{HO}(\mathrm{Thr} 108)$ & 1.799 \\
\hline & & & & $\mathrm{CO} \cdots \mathrm{HN}($ Ala109) & 1.840 \\
\hline \multirow{2}{*}{$6 b$} & \multirow{2}{*}{2} & \multirow{2}{*}{-6.35} & \multirow{2}{*}{1.457} & $\mathrm{CO} \cdots \mathrm{HN}(\mathrm{Lys} 117)$ & 2.019 \\
\hline & & & & $\mathrm{CO} \cdots \mathrm{HN}(\mathrm{Asn} 118)$ & 1.866 \\
\hline
\end{tabular}

Ntor, the number of torsional degree of freedom in the compound; $\Delta G_{A D 4}$, the predicted free energy of binding from AutoDock4; RMSD, the root mean square difference in coordinates between the atoms and docked conformation. 
Table 3a. PASS predictions of antibiotic Penicillin-like compounds with physical properties

\begin{tabular}{|c|c|c|c|c|}
\hline NSC No. & 2D structure & No. H-bond acceptors & No. H-bond donors & No. rotatable bonds \\
\hline 126846 & & 4 & 1 & 5 \\
\hline 193396 & & 4 & 2 & 4 \\
\hline 277174 & & 6 & 4 & 4 \\
\hline
\end{tabular}

Table 3b. PASS prediction of $\beta$-lactamases inhibitor compound with physical properties

\begin{tabular}{llll}
\hline NSC No. & No. H-bond acceptors & No. H-bond donors No. rotatable bonds \\
\hline 22409 & $2 D$ structure & 3 & 0 \\
\end{tabular}

ed NCI Database Browser for "penicillin-like antibiotics" which are structurally similar to compound $\mathbf{5 b}$. Since penicillin-like antibiotics are substrates for M $\beta \mathrm{Ls}$, it is known that their chemical structure allows binding to the active site. From this NCI database screening, it was expected that chemical moieties could be identified which would allow a $\mathbf{5 b}$-like inhibitor to bind closer to the active site, thereby improving inhibition. We also conducted a PASS search for "beta-lactamase inhibitors" because a previous study suggested that some compounds can inhibit both serine-based $\beta$-lactamases and M $\beta$ Ls. ${ }^{23}$ The four compounds shown in Table $3 \mathrm{a}$ and $3 \mathrm{~b}$ were selected out of 250,251 Open NCI Database structures. The PASS prediction query for "penicillin-like antibiotics" found three compounds
126846, 193396, and 277174 and a query for "beta-lactamase inhibitors" found compound 222409, where 193396 and 277174 are Penicillin G and Amoxicillin, respectively. AutoDock 4.0 was used to dock these compounds with di-zinc $\mathrm{M} \beta \mathrm{L}$ from Bacillus cereus. For compound 126846, the lowest predicted binding energy was $-7.20 \mathrm{kcal} / \mathrm{mol}$ with four hydrogen bond interactions. The interactions were present between the $\beta$-lactam carboxylate moiety of 126846 and the amine hydrogen of Ala89 $(1.940 \AA)$, the side chain oxygen of 126846 and the amine hydrogen of Lys $117(1.866 \AA)$, the side chain amine hydrogen of 126846 and the carbonyl oxygen of $\operatorname{Asn} 118$ (2.217 $\AA$ ), and the carbonyl oxygen of 126846 's $\beta$-lactam ring and the amine hydrogen of Asn118 (2.155 $\AA$ ). For compound 193396, the 


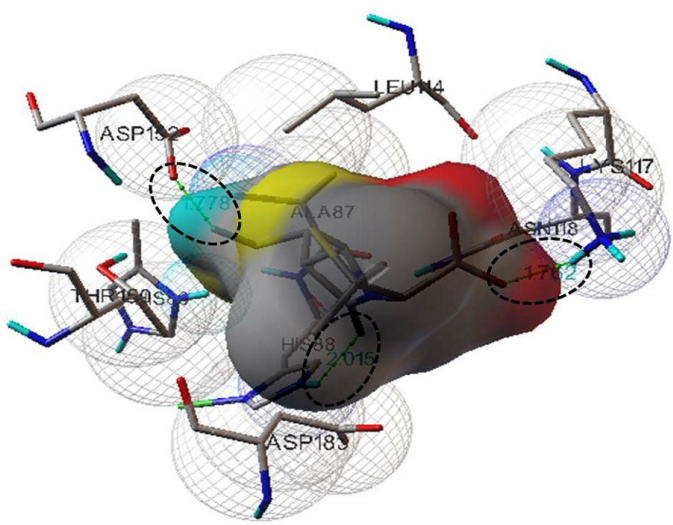

Figure 3. Docking interactions of compound $\mathbf{5 b}$; spheres as wireframe indicate atoms in close contact, compound $\mathrm{msms}$ are displayed as sold cloud, and hydrogen bonding interaction as dash lines are circled with distance.

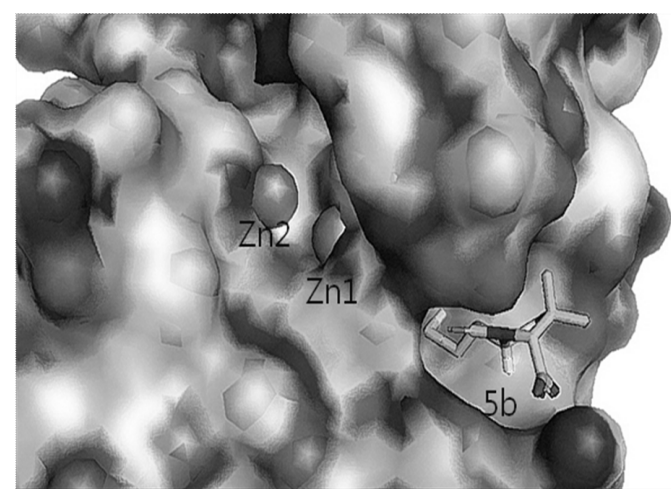

Figure 4. Molecular surface map of the Bacillus cereus with compound 5 b.

Table 4. Docking results with NCI Database

\begin{tabular}{|c|c|c|c|c|c|}
\hline Compound (NSC No.) & Ntor & $\Delta \mathrm{G}_{\mathrm{AD} 4}(\mathrm{kcal} / \mathrm{mol})$ & $\operatorname{RMSD}(\AA)$ & H-bonding interaction & H-bond distance $(\AA)$ \\
\hline 126846 & 5 & -7.20 & 7.406 & 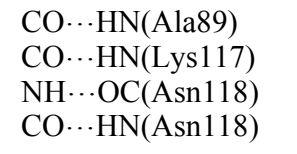 & $\begin{array}{l}1.940 \\
1.866 \\
2.217 \\
2.155\end{array}$ \\
\hline 193396 & 4 & -7.17 & 1.057 & $\mathrm{CO} \cdots \mathrm{HN}(\mathrm{ASP} 90)$ & 1.947 \\
\hline 277174 & 4 & -7.96 & 5.513 & 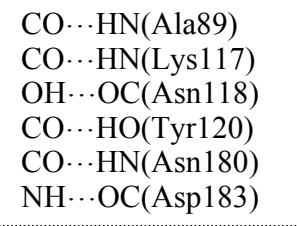 & $\begin{array}{l}1.738 \\
1.792 \\
1.904 \\
1.634 \\
2.061 \\
1.736\end{array}$ \\
\hline 222409 & 2 & -6.84 & 1.175 & $\mathrm{CN} \cdots \mathrm{HN}(\mathrm{Lys} 117)$ & 1.965 \\
\hline
\end{tabular}

Ntor, the number of torsional degree of freedom in the compound; $\Delta \mathrm{G}_{\mathrm{AD} 4}$, the predicted free energy of binding from AutoDock4; RMSD, the root mean square difference in coordinates between the atoms and docked conformation.

lowest predicted binding energy was $-7.17 \mathrm{kcal} / \mathrm{mol}$. In this binding conformation, there was one hydrogen bond interaction for 193396: the carbonyl oxygen of the $\beta$-lactam 6-acylamino side chain and the amine hydrogen of Asp90 with a distance of $1.947 \AA$ A. Docking simulations of the binding of 193396 to monozinc $\beta$-lactamases from Bacillus cereus have previously been performed; the results of these studies suggest that Lys 171 and Asn180, residues conserved in all known MBLs, are involved in hydrogen bond formation with $193396 .^{22,27,28}$ In our docking experiment, secondary docking conformations of 193396 revealed a hydrogen bond between its $\beta$-lactam carboxylate moiety and the conserved residues, Lys 171 and Asn 180 (data not shown), which is in accordance with the findings of the previous studies. For compound 277174, the lowest predicted binding energy was $-7.96 \mathrm{kcal} / \mathrm{mol}$ with six hydrogen bond interactions. The interactions were present between the $\beta$ lactam carboxylate moiety of 277174 and the amine hydrogen of Ala89 (1.738 $\AA$ ), side chain oxygen of 277174 and the amine hydrogen of Lys 117 (1.792 $\AA$ ), hydroxyl on the aromatic side chain of 277174 and the amine hydrogen of Asn118 (1.904 $\AA$ ), $\beta$-lactam carboxylate moiety of 277174 and side chain hydroxyl of Tyr120 (1.634 А), carbonyl oxygen of 277174's amide and the amine hydrogen of Asn 180 (2.061 $\AA$ ), and finally, the side chain amine hydrogen of 277174 and the carbonyl oxygen of Asp183 (1.736 A). In the case of compound 222409, although the structure does not possess a $\beta$-lactam ring like the others, the pass prediction suggested that it possesses strong predicted activity as a $\beta$-lactamase inhibitor. The results of the docking experiments showed a lowest predicted binding energy of -6.84 $\mathrm{kcal} / \mathrm{mol}$. A hydrogen bond occurred between the nitrogen-containing five-membered ring of 222409 and the amine hydrogen of Lys 117 (1.965 $\AA$ ). However, there is no evidence that Lys 117 is critical to the M $\beta \mathrm{L}$ mechanism, suggesting that 222409 may not be a suitable candidate for further investigation. The all docking results are illustrated in Table 4.

The docking results with the NCI compounds showed that 277174 had a lowest predicted binding energy and six hydrogen bonding interactions, thus 277174 initially appeared to be the best potential inhibitory compound for the M $\beta L$. However, the residues responsible for the hydrogen bonding interactions do not participate in the enzyme catalysis. Unlike 277174, the docking results with 193396 showed a hydrogen bonding interaction 

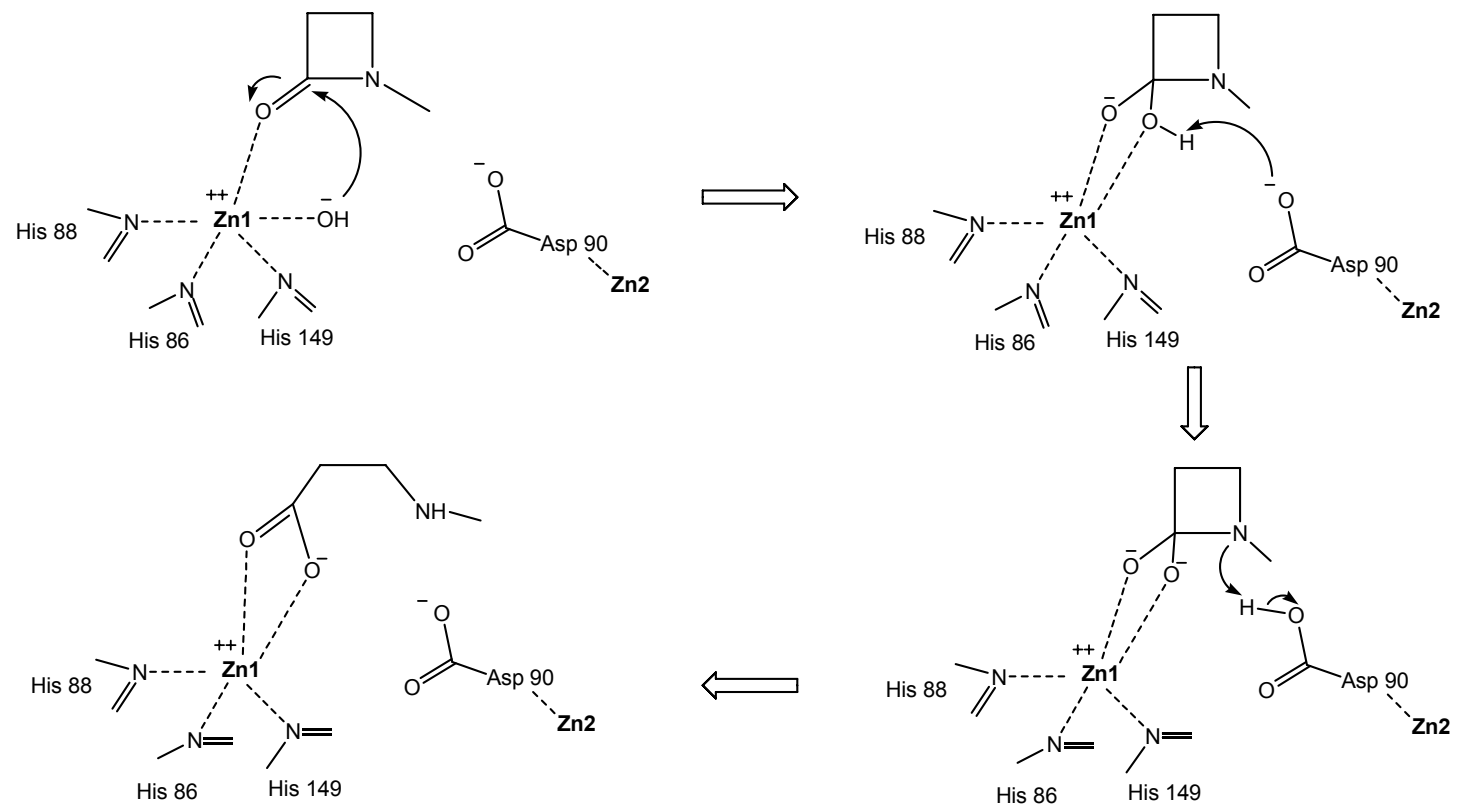

Figure 5. Proposed mechanism of $\beta$-lactam ring hydrolysis (adopted from ref $\#^{25}$ ).<smiles>CC1(C)S[C@@H]2[C@H](CC(=O)Cc3ccccc3)C(=O)N2[C@H]1C(=O)O</smiles>

A<smiles>CC1(C)S[C@@H]2[C@H](NC(=O)Cc3ccccc3)C(=O)N2[C@H]1C(=O)O</smiles>

B<smiles>C[C@]1(CC(=O)Cc2ccccc2)S[C@@H]2[C@H](CS)C(=O)N2[C@H]1C(=O)O</smiles>

C<smiles>C[C@]1(CC(=O)Cc2ccccc2)S[C@@H]2[C@@H](CS)C(=O)N2[C@H]1C(=O)O</smiles>

D<smiles>CC1(C)S[C@@H]2[C@@H](CSOC(=O)[C@@H]3N4C(=O)[C@@H](NC(=O)Cc5ccccc5)C4S3(C)C)C(=O)N2[C@H]1C(=O)O</smiles>

E

Figure 6. Hypothetically designed M $\beta$ Ls inhibitor compounds.

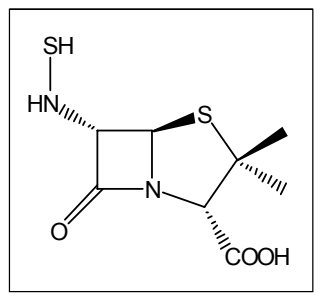

F

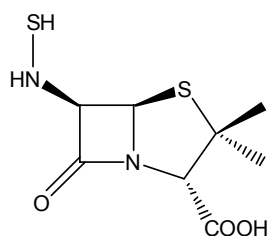

G with the active site residue Asp90, whose carboxylate anion plays a significant role in amide hydrolysis of the lactam ring. ${ }^{25,29}$ (Figure 5). Although the hydrogen bonding with Asp90 occurred between the $\beta$-lactam 6-acylamino side chain and the amine hydrogen of Asp90 instead of its carboxyl side chain, 193396 still binds closer to the active site than the other compounds. For this reason, compound 193396 was chosen as a basis for several hypothetical inhibitor designs.

Predicted inhibitor design. Docking results from four predefined compounds and four compounds obtained from the NCI database suggest that both $\mathbf{5 b}$ and 193396 interact with the active site of the target protein $\mathrm{M} \beta \mathrm{L}$ from Bacillus cereus. Although compound $\mathbf{5 b}$ showed significant inhibition, it does not seem to directly occlude active site access by a substrate, as shown in the binding topology diagram in Figure 4. Compound 193396, alternatively, binds to Asp90, which is part of the Zn2 active site, but it interacts with the amine hydrogen of Asp90 instead of the carboxyl side chain, allowing the M $\beta$ Ls to hydrolyze the $\beta$-lactam ring of 193396 . By combining certain aspects of compounds $\mathbf{5} \mathbf{b}$ and $\mathbf{1 9 3 3 9 6}$, there is a possibility that the inhibitor design can be improved. To acquire more effective potential inhibitors, we designed hypothetical compounds based 
Table 5. The best docking results of suggested compound

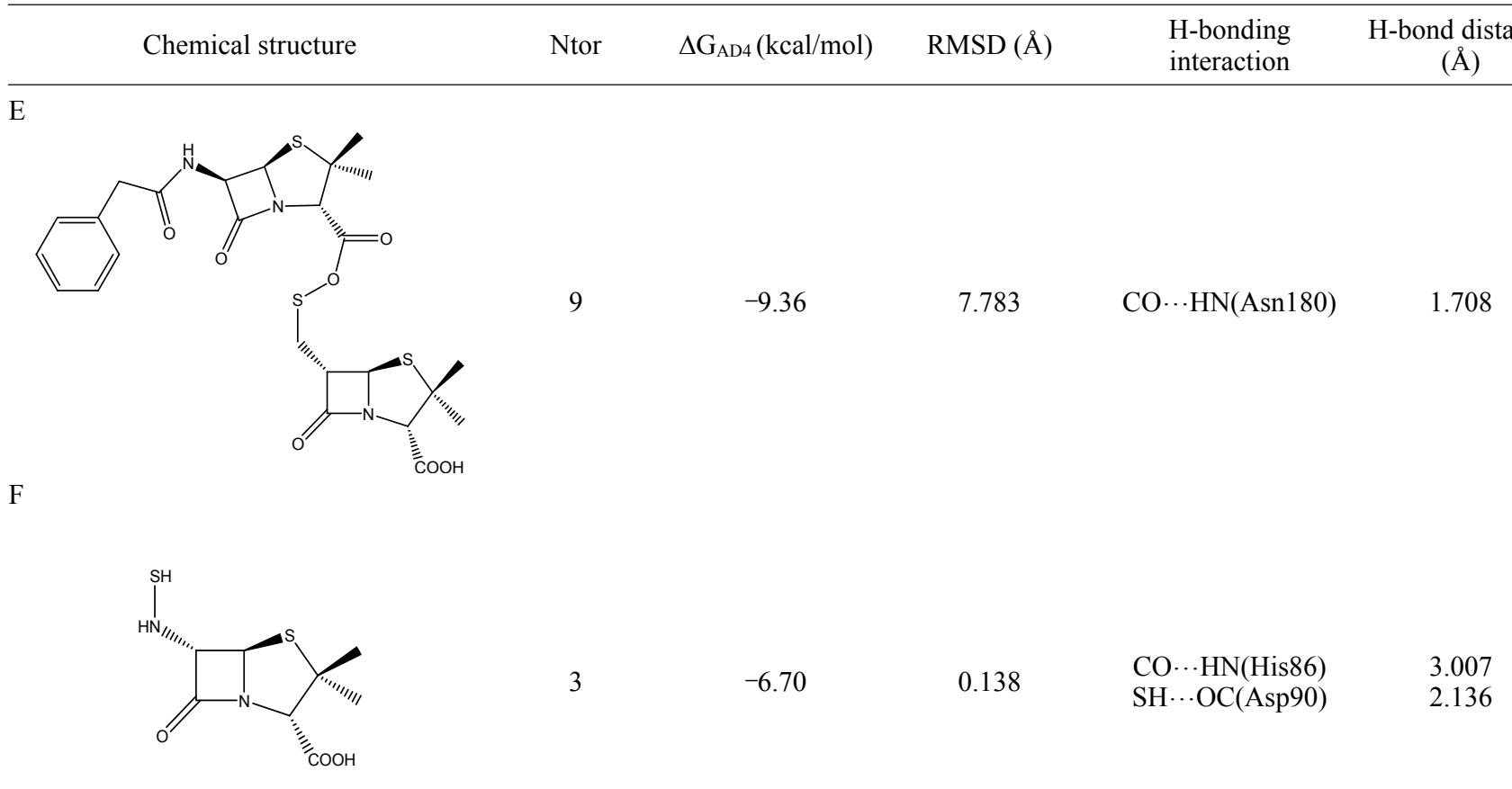

Ntor, the number of torsional degree of freedom in the compound; $\Delta \mathrm{G}_{\mathrm{AD} 4}$, the predicted free energy of binding from AutoDock4; RMSD, the root mean square difference in coordinates between the atoms and docked conformation.

on the structures of $\mathbf{5 b}$ and 193396 (Figure 6) and then docked these hypothetical compounds with the M $\beta L$. The goal of this investigation was to discover compounds which bind near both zinc1 and zinc2 containing active sites. Hydrogen bonding interactions with the active site residues and binding energy are both important factors when evaluating the inhibitor candidates. By tightly binding near the $\mathrm{Zn} 1$ and $\mathrm{Zn} 2$ containing active sites, an inhibitor could disrupt certain critical hydrogen bonds, reducing the stability of the $\mathrm{Zn} 1$ tetrahedral intermediate and decreasing the basicity of Asp90. ${ }^{25}$

The designs of all novel structures in Figure 6 were based upon compounds $\mathbf{5 b}$ and 193396. Because the scaffolds of compounds $\mathbf{5 b}$ and 193396 are similar, the functional groups of these compounds were exchanged with stereochemical variation, generating compounds $\mathrm{A}, \mathrm{F}$, and $\mathrm{G}$. Compound $\mathrm{B}$ is a stereochemical variation of 193396 while compound $\mathrm{E}$ was generated by the combination of $\mathbf{5} \mathbf{b}$ and 193396, in their entirety, via an S-O bond. Lastly, compounds $\mathrm{C}$ and $\mathrm{D}$ were generated by attaching the side chain of 193396 to the thiazolidine ring of $\mathbf{5 b}$ in different stereochemical arrangements. Docking experiments revealed that two of the seven hypothetical compounds (i.e., compounds $\mathrm{E}$ and $\mathrm{F}$ ) bind to the $\mathrm{Zn} 1$ and $\mathrm{Zn} 2$ containing active sites with reasonably low binding energies (Table 5).

Compound $\mathrm{E}$, a molecule designed by connecting compounds 5b and 193396 with an S-O bond, had a lowest predicted binding energy of $-9.36 \mathrm{kcal} / \mathrm{mol}$. This binding energy is lower than penicillin-like antibiotics such as penicillin $\mathrm{G}$ and Amoxicillin, and thus $\mathrm{E}$ appears to be a potential $\mathrm{M} \beta \mathrm{L}$ inhibitor that may compete with penicillin-like antibiotics. The compound's large size allows for many interactions with the protein, thereby contributing to its low binding energy. In addition to the low binding energy, a hydrogen bonding interaction between the $\beta$ lactam carbonyl of compound $\mathrm{E}$ and the Asn 180 side chain was observed (Table 5). Since Asn1 180 is a conserved residue, it is likely that Asn 180 helps to orient potential substrates into the binding pocket. This interaction may impede substrate binding with the active sites. Hence, E initially seems to be a promising $\mathrm{M} \beta \mathrm{L}$ inhibitor candidate.

Compound $\mathrm{F}$, a variation of compound $\mathbf{5 b}$ in which the thiol group is connected to the $\beta$-lactam ring through a nitrogen instead of carbon, had a lowest predicted binding energy of -6.70 $\mathrm{kcal} / \mathrm{mol}$. As shown in Figure 7a, two hydrogen bonding interactions were found between the carbonyl oxygen of compound F's $\beta$-lactam ring and the His86 side chain (3.007 $\AA$ ) and between the thiol hydrogen of compound $F$ and the Asp90 side chain (2.136 А). Both His86 and Asp90 are coordinated to Zn1 and $\mathrm{Zn} 2$, respectively. These residues are critical to the proposed mechanism of the M $\beta \mathrm{L}$ (Figure 5). His 86 provides stability to the $\mathrm{Zn} 1$ tetrahedral arrangement while Asp90 acts as a general base, catalyzing the hydrolysis of the lactam ring. Compound $\mathrm{F}$ could therefore possibly serve as an obstacle to the zinc1 electrophilic attack of the $\beta$-lactam carbonyl oxygen and hinder the breakdown of the $\mathrm{C}-\mathrm{N}$ bond in the $\beta$-lactam ring. Furthermore, by hydrogen bonding to both these residues, compound $\mathrm{F}$ may possess enhanced stability and selectivity.

The results indicate that both hypothetical compounds $\mathrm{E}$ and F possess improved topological hindrances and binding energies compared to the previously suggested compound $\mathbf{5 b}$. Although compound $\mathrm{E}$ possesses a lower binding energy than penicillinlike antibiotics, E, because it is so large and structurally com- 
(a)

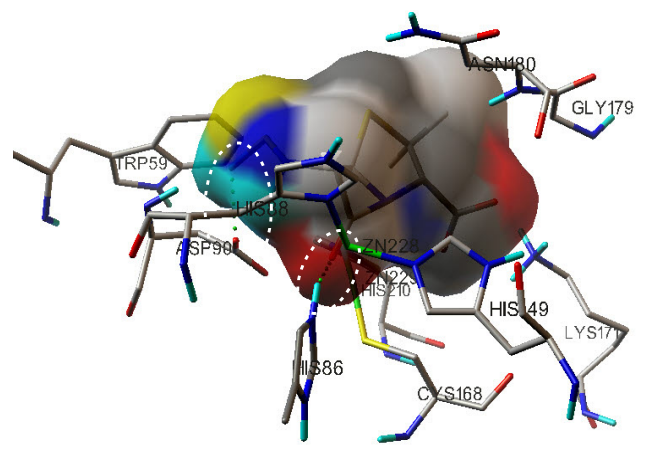

(b)

(c)
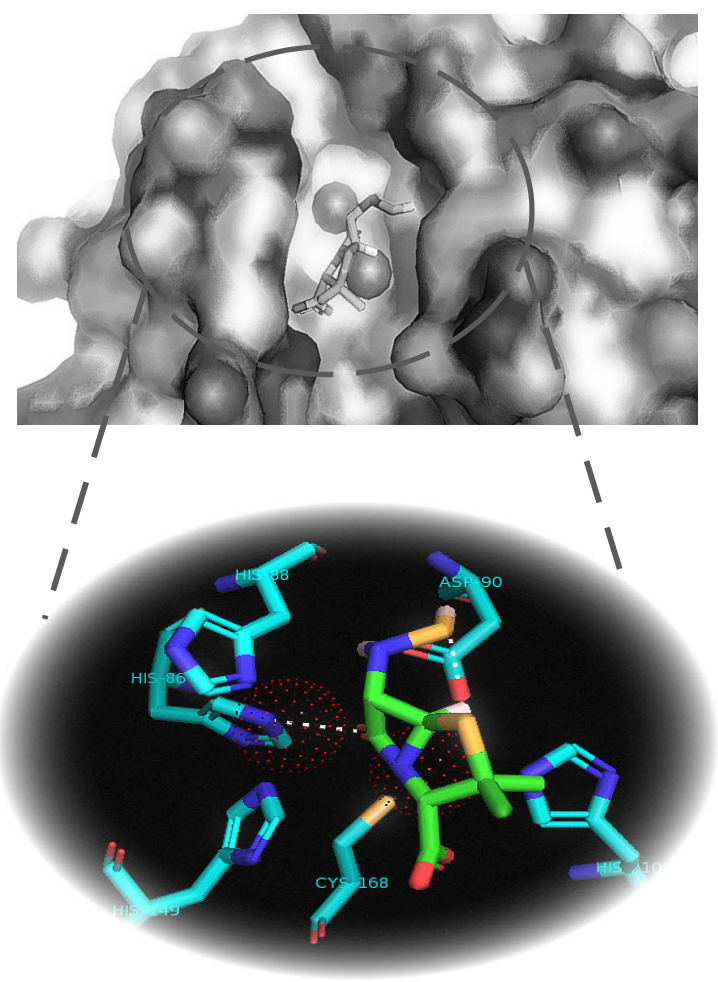

Figure 7. Docking interactions of suggested compound F. (a) Docking interactions of compound F; spheres as wireframe indicate atoms in close contact, compound MSMS are displayed as solid cloud, and hydrogen bonding interaction as dash lines are circled. (b) A surface diagram of docked suggested compound $\mathrm{F}$ to the active site binding pocket of the Bacillus cereus and (c) Docking interaction between zinc coordinated residues and suggested compound $\mathrm{F}$.

plex, appears to be unstable. In addition, the CAS (Chemical Abstracts Service) registry does not possess an example of the $\mathrm{S}-\mathrm{O}$ bond contained in this compound. Therefore, a practical laboratory synthesis of this compound is questionable. On the other hand, because compound $\mathrm{F}$ is more structurally stable, smaller, and has more hydrogen bonding interactions with the active site than $\mathrm{E}$, the data show that $\mathrm{F}$ is more likely to fit into the binding pocket (Figure $7 \mathrm{~b}$ and $7 \mathrm{c}$ ). Also, the CAS registry contains several compounds (CAS registry numbers: 66788451-7 and 733710-18-4) with similar mercaptoamine bonds (SH$\mathrm{NH}$ ) which will provide practical information for the synthesis of compound $\mathrm{F}$. Thus, the results from docking experiments suggest that compound $\mathrm{F}$ is a more promising $\mathrm{M} \beta \mathrm{L}$ inhibitor design.

\section{Conclusion}

The main goal of this study was to build upon a previous in vitro study of $\mathrm{M} \beta \mathrm{Ls}$ inhibitor compounds and, through computational screening, generate a more effective potential inhibitor design which maintains a penicillin-like scaffold. We applied virtual screening, using AutoDock4.0, to a total of eight predefined and NCI database compounds in order to identify specific structural characteristics which encourage inhibition. We then sought to exploit these characteristics and combine them into a single, hypothetical inhibitor design. By using AutoDock4.0, we were able to expand on the results of previous in vitro studies and use these findings to propose a novel potential $\mathrm{M} \beta \mathrm{L}$ inhibitor, compound F. Further investigations into our suggested compound may provide practical information and potent motivation for organic chemists seeking to synthesize a new $\mathrm{M} \beta \mathrm{L}$ inhibitor compound.

Acknowledgments. This work was funded by grants from the Young Investigator Development Program at Baylor University. Authors would like to thank Patricia Zhang and Joongwon Lee for their invaluable technical assistance and unfailing editorial advice.

\section{References}

1. Fisher, J. F.; Meroueh, S. O.; Mobashery, S. Chem. Rev. 2005, $105(2), 395$.

2. Abriata, L. A.; Gonzalez, L. J.; Llarrull, L. I.; Tomatis, P. E.; Myers, W. K.; Costello, A. L.; Tierney, D. L.; Vila, A. J. Biochemistry 2008, 47(33), 8590.

3. Heinz, U.; Adolph, H. W. Cell. Mol. Life Sci. 2004, 61(22), 2827.

4. Sykes, R. B.; Matthew, M. J. Antimicrob. Chemother. 1976, 2(2), 115.

5. Medeiros, A. A. Br. Med. Bull. 1984, 40(1), 18.

6. Matagne, A.; Dubus, A.; Galleni, M.; Frere, J. M. Nat. Prod. Rep. 1999, $16(1), 1$.

7. Frere, J. M.; Dubus, A.; Galleni, M.; Matagne, A.; Amicosante, G. Biochem. Soc. Trans. 1999, 27(2), 58.

8. Page, M. I. Curr Pharm Des 1999, 5(11), 895

9. Hu, Z.; Periyannan, G. R.; Crowder, M. W. Anal. Biochem. 2008, 378(2), 177.

10. Jacquin, O.; Balbeur, D.; Damblon, C.; Marchot, P.; Pauw, E. De.; Roberts, G. C.; Frere, J. M.; Matagne, A. J. Mol. Biol. 2009, 392(5), 1278.

11. Wang, Z.; Fast, W.; Valentine, A. M.; Benkovic, S. J. Curr. Opin. Chem. Biol. 1999, 3(5), 614.

12. Payne, D. J.; Bateson, J. H.; Gasson, B. C.; Proctor, D.; Khushi, T.; Farmer, T. H.; Tolson, D. A.; Bell, D.; Skett, P. W.; Marshall, A. C.; Reid, R.; Ghosez, L.; Combret, Y.; Marchand-Brynaert, J. Antimicrob. Agents. Chemother. 1997, 41(1), 135.

13. Simm, A. M.; Loveridge, E. J.; Crosby, J.; Avison, M. B.; Walsh, T. R.; Bennett, P. M. Biochem. J. 2005, 387(Pt 3), 585.

14. Kapetanovic, I. M. Chem. Biol. Interact. 2008, 171(2), 165.

15. Pozzan, A. Curr. Pharm. Des. 2006, 12(17), 2099.

16. Green, D. V. Prog. Med. Chem. 2003, 41, 61.

17. Chen, D.; Misra, M.; Sower, L.; Peterson, J. W.; Kellogg, G. E.; Schein, C. H. Bioorg. Med. Chem. 2008, 16(15), 7225.

18. Jaganatharaja, J.; Gowthaman, R. Bioinformation 2006, 1(4), 112.

19. Rogers, J. P.; Beuscher, A. E. t.; Flajolet, M.; McAvoy, T.; Nairn, A. C.; Olson, A. J.; Greengard, P. J. Med. Chem. 2006, 49(5), 1658.

20. Cosconati, S.; Hong, J. A.; Novellino, E.; Carroll, K. S.; Goodsell, D. S.; Olson, A. J. J. Med. Chem. 2008, 51(21), 6627. 
21. Huey, R.; Morris, G. M.; Olson, A. J.; Goodsell, D. S. J. Comput. Chem. 2007, 28(6), 1145.

22. Fabiane, S. M.; Sohi, M. K.; Wan, T.; Payne, D. J.; Bateson, J. H.; Mitchell, T.; Sutton, B. J. Biochemistry 1998, 37(36), 12404.

23. Buynak, J. D.; Chen, H.; Vogeti, L.; Gadhachanda, V. R.; Buchanan, C. A.; Palzkill, T.; Shaw, R. W.; Spencer, J.; Walsh, T. R. Bioorg Med. Chem. Lett. 2004, 14(5), 1299.

24. Schuttelkopf, A. W.; van Aalten, D. M. Acta Crystallogr. D. Biol. Crystallogr. 2004, 60(Pt 8), 1355.
25. Bounaga, S.; Laws, A. P.; Galleni, M.; Page, M. I. J. Biochem. 1998, 331 (Pt 3), 703.

26. Kim, S. K. Ph.D. dissertation, Texas Tech Universtiy, USA 2002.

27. Diaz, N.; Suarez, D.; Merz, K. M. Jr. J. Am. Chem. Soc. 2001, 123(40), 9867.

28. Prosperi-Meys, C.; Wouters, J.; Galleni, M.; Lamotte-Brasseur, J. Cell Mol. Life Sci. 2001, 58(14), 2136.

29. Gensmantel, N. P.; Proctor, P.; Page, M. I. J.C.S. Perkin 1980, II, 1725. 